\title{
To the Editor: Predictive Markers and Precision Medicine
}

The authors of the recently published point and counterpoint commentaries on precision medicine ${ }^{1,2}$ agreed that the success of precision medicine is largely predicated on the identification of highly predictive markers of efficacy, but they do not discuss 2 fundamental aspects of personalized, precision oncology. First, expanding the definition of a particular predictive marker without clinical trials to support that change can be problematic. Second, more sensitive predictive markers should not distract from recognizing that the values, hopes, and dreams of patients remain the fundamental basis for why they choose either to undergo therapy or to forego even a highly effective targeted therapy.

For example, so remarkable was the benefit of trastuzumab combined with chemotherapy over chemotherapy alone for prolonging survival or reducing the likelihood of recurrence after surgery, that in 2013 the expert ASCO/CAP panel (and later the NCCN Breast Cancer Panel) expanded the 2007 definition of HER2-positive breast cancer to include patients who would now be identified as having "HER2-positive" disease based only on metrics such as identifying HER2 gene amplification using "alternative probes" or using polysomy as a surrogate marker of HER2 positivity., Although the panel did so with the hope of making highly effective anti-HER2 therapies available to more patients (ie, so that "the right patient receives the right treatment"), they acknowledged that they did so with very few clinical data. Confirmatory studies of the efficacy of anti-HER2 therapy in patients with HER2-positive tumors based only on one of the new 2013 criteria was understandably problematic once the endorsement of the new definitions by the ASCO/CAP panel and NCCN had occurred.

The heart of personalized or precision medicine will always be how the patient views his or her options. As William Osler said, "it is much more important to know what sort of patient has a disease than what sort of disease a patient has." The remarkable targeted therapies and the markers that predict their efficacy must be placed in the context of how the patient feels about the risks and benefits. No assay will ever quantify how a particular patient feels about enduring what may be a toxic drug to potentially enjoy one more sunny spring morning, sharing laughter with a friend, being there for the graduation of a child, or listening to a beautiful symphony. Understanding the patient's essence remains fundamental to personalized, precision oncology.

Steven Sorscher, MD

Wake Forest School of Medicine

\section{References}

1. Gyawali B. Point: the imprecise pursuit of precision medicine: are biomarkers to blame? J Natl Compr Canc Netw 2017;15:859-860

2. Kato S, Subbiah V, Kurzrock R. Counterpoint: success in the pursuit of precision medicine: biomarkers take credit. J Natl Compr Canc Netw 2017;15:863-866.

3. Wolff AC, Hammond EH, Hicks DG, et al. Recommendations for human epidermal growth factor receptor 2 testing in breast cancer: American Society of Clinical Oncology/College of American Pathologists clinical practice guideline update. J Clin Oncol 2013;31:3997-4013.

4. Hanna WM, Slodkowska E, Houman N, et al. Comparative analysis of human epidermal growth factor receptor 2 testing in breast cancer according to 2007 and 2013 American Society of Clinical Oncology/College of American Pathologists Guideline recommendations. J Clin Oncol 2017;35:3039-3045. 